\title{
Repetitive Observer Design for Torque Ripple Reduction in PMSM Drives
}

\author{
Mi Tang, Andrea Formentini, Pericle Zanchetta \\ UNVIERSITY OF NOTTINGHAM \\ University Park \\ Nottingham, UK \\ E-Mail: mi.tang2@nottingham.ac.uk
}

\section{Acknowledgements}

This work is financially supported by Shenzhen Best Motion Technology Ltd.

\section{Keywords}

« Control of drive », « Control methods for electrical systems», « Design», « Harmonics »

\begin{abstract}
Control strategies aimed at the suppression of periodic disturbance, such as Repetitive Controller (RC) and disturbance observer (DO) have been used in many applications in the past few years. A traditional RC and a conventional DO can be merged into a novel repetitive observer (RO). This paper provides tools to design a RO and proves mathematically that the separation principle used in the DO design is also valid for the RO. The effects of each control parameter on the system performance are also studied and the RO approach is then tested for torque ripple reduction in permanent magnet synchronous machine (PMSM) drives.
\end{abstract}

\section{Introduction}

Repetitive controller (RC) has received increasing interest in the past years as an effective solution for rejecting periodic disturbances in closed loop control systems for power electronics and drives applications. In power electronics, periodic disturbances are, for example, harmonics on the power supply or generated by non-linear loads [1]. In drive systems, a periodic disturbance can be represented by the torque ripple [2] produced by cogging effects, non-sinusoidal flux distribution and any mechanical misalignment.

$\mathrm{RC}$ works only on the harmonics suppression to supplement a conventional feedback controller. One of the main advantages of RC is that it can be designed by only knowing the frequency of the disturbance. However, due to lack of information about the plant, the design of the controller can be challenging in terms of stability. For example, some main causes of instability can be parameter variations of the controlled plant, which modify gain and phase of the whole system; or variation of the plant delays, which consequently causes unwanted phase shift between reference and controlled variable.

Since harmonics can be modelled as disturbances, a disturbance-observer (DO) can also be used as a supplement to the feedback controller for harmonic cancellation as in[3-6].

On one hand, the main benefit of choosing the DO is that it can be added to an existing control loop directly without affecting the system stability, because the separation principle can be applied [7]. Usually, there is a low-pass filter in the DO to attenuate measurement noise. If the frequency of the target disturbance is close to the measurement noise, the performance of DO will degrade[5]. On the other hand, the RC has stronger noise rejection ability since a high order filter with a sharp cut-off characteristic can be used. However, the $\mathrm{RC}$ is dynamically much slower than the DO. It requires at least one disturbance fundamental period of delay before it takes effect. Moreover, it needs to be designed together with the feedback controller to ensure the system stability. 
Taking benefits of the best characteristics of both methods, authors of $[7,8]$ have pointed out that by adding a correction term in $\mathrm{RC}$ and considering the disturbance as states, the resulting $\mathrm{RC}$ can be designed as a DO. This new hybrid approach has been applied to grid-connected voltage source inverters for reducing the output voltage Total Harmonic Distortion (THD) in [9] and is named as Repetitive Observer (RO) in [8] for torque ripple reduction in permanent magnet synchronous machine (PMSM) drives. It has been claimed in [7] that the RO performs like a traditional RC for harmonics attenuation, but a larger stability margin can be achieved without sacrificing its performance. In addition, it is claimed in [8] that RO can be designed using the same method as a conventional DO. However, no rigorous theoretical proof and design guidelines have been yet provided in order to support the method. Moreover, the effects of the control parameters of the RO on its disturbance rejection ability are still not clear.

Therefore, this paper aims to provide clear design guidelines for a $\mathrm{RO}$ and prove mathematically that the separation principle used in the DO design can also be applied in the design of a RO, which is then tested for improving torque ripple reduction capabilities in PMSM drives. The relationship between each control parameters in the RO and its disturbance suppression performance is also studied. Moreover, although the feedback controller and the RO are independent from the stability point of view, their interactions are analysed to find the combination that provides the best performance.

The paper will be arranged as follows: Section II will describe and compare traditional Proportional-Integral (PI) control loops with the insertion of $\mathrm{RC}, \mathrm{DO}$ and $\mathrm{RO}$ respectively. The separation principle for the $\mathrm{RO}$ will be proven in Section III. The effects of each control parameter in the RO on the performance will be discussed in the simulation results of Section IV and design guidelines will be provided. Experimental validation of the simulation results will be given in Section $\mathrm{V}$ for the case of torque ripple reduction in a PMSM drives, while conclusions will be shown in Section VI.

\section{RO plus PI control loop}

Fig. 1 shows the block diagram considering the application of a RO approach in support of a PI speed closed loop control for the torque ripple reduction in a PMSM drive, where, $R$ denotes the control reference and $E$ denotes the control error. The proportional and integral gains for the PI controller are $K_{p}$ and $K_{i}$, respectively. $U$ is the input of the plant, the output of the plant is the state $V$, which equals $x_{2}$. The plant $P$ is assumed to be a simple first order mechanical system, of which the continuous transfer function is $1 /\left(J_{S}+B\right)$. The fundamental frequency of the periodic disturbance $D$ (torque ripple) is $f_{d}$, whereas the sampling frequency is $f_{s}$. Therefore, for every $N=f_{s} / f_{d}$ samples, the disturbance repeats itself. In this paper, the RO as shown in Fig. 1 is used for cancelling the torque ripple $D$. Fig.2a and Fig.2b show instead, the system diagrams with only $\mathrm{RC}$ and DO, respectively.

The parameters of the RO in Fig.1 will be given in details in the next section. In general, on one hand, the high dimension matrices (which have been highlighted in bold) in the RO provide the same effect as the long delay chain $z^{-N}$ in the RC. The parameter $Q$ (will be defined in the next section) in the RC has also been included in the matrix $\overline{\boldsymbol{A}_{d}}$. On the other hand, the RO has the same structure as the DO, although the dimension of the DO is much lower (i.e. $1^{\text {st }}$ order).

\section{Proof of separation principle}

According to Fig.1, the discretised form of the plant transfer function can be expressed as in (1).

$$
\mathrm{P}(\mathrm{z})=\frac{x_{2}(z)}{U(z)+D(z)}=\frac{z-1}{z} \mathbb{Z}\left\{\frac{1}{s(J s+B)}\right\}=\frac{b_{1}}{z-a_{1}}, a_{1}=e^{-\frac{B}{J} T}, b_{1}=\frac{1-e^{-\frac{B}{J} T}}{B}
$$

where, $T$ denotes the sampling period. 

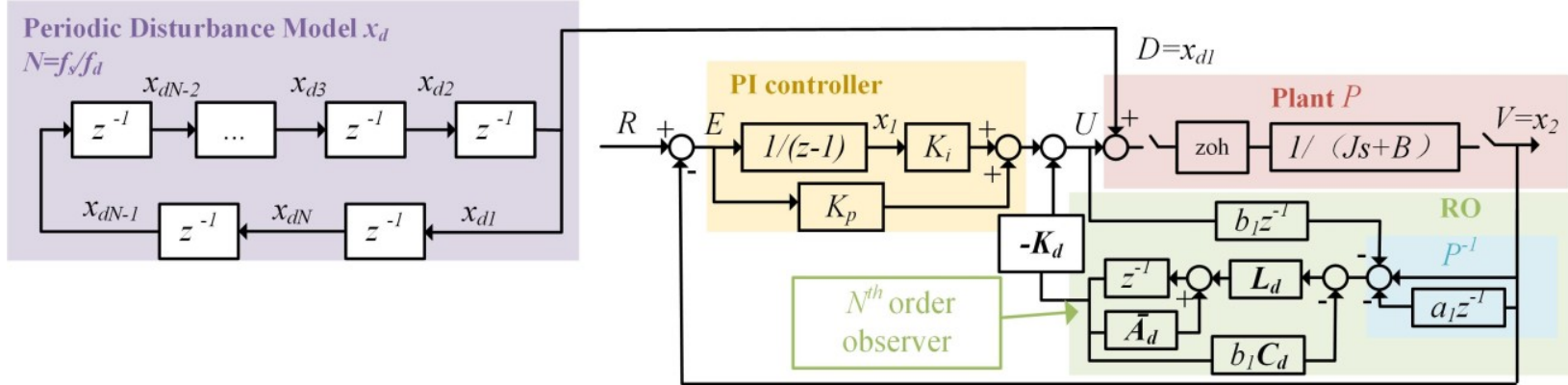

Fig.1 Block diagram of a typical PI control loop with RO.

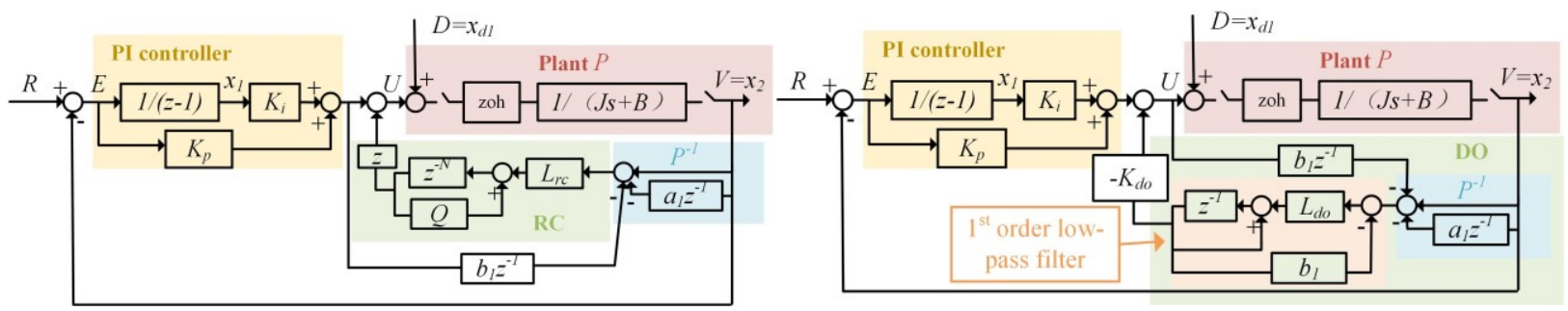

(a)

(b)

Fig.2 Block diagram of a typical PI control loop with RC and DO.

From (1), the state-space equation of the control loop in Fig.1 can be derived as in (2):

$$
\left\{\begin{array}{c}
\boldsymbol{x}(k+1)=\boldsymbol{A} \boldsymbol{x}(k)+\boldsymbol{B} R(k)+\boldsymbol{E} D(k) \\
V(k)=\boldsymbol{C} \boldsymbol{x}(k)
\end{array}\right.
$$

where, $\boldsymbol{x}(k)=\left[\begin{array}{l}x_{1}(k) \\ x_{2}(k)\end{array}\right], \boldsymbol{A}=\left[\begin{array}{cc}1 & -1 \\ b_{1} k_{i} & a_{1}-b_{1} k_{p}\end{array}\right], \mathbf{B}=\left[\begin{array}{c}1 \\ b_{1} k_{p}\end{array}\right], \mathbf{C}=\left[\begin{array}{ll}0 & 1\end{array}\right], \mathbf{E}=\left[\begin{array}{c}0 \\ b_{1}\end{array}\right]$.

Again, assuming the fundamental frequency of the periodic disturbance is $f_{d}$, and define $N=f_{s} / f_{d}$, the disturbance can therefore be modelled using the $N$ states as in (3).

$$
\left\{\begin{array}{c}
\boldsymbol{x}_{\boldsymbol{d}}(k+1)=\boldsymbol{A}_{\boldsymbol{d}} \boldsymbol{x}_{\boldsymbol{d}}(k) \\
D(k)=\boldsymbol{C}_{\boldsymbol{d}} \boldsymbol{x}_{\boldsymbol{d}}(k)
\end{array}\right.
$$

where, $\boldsymbol{x}_{\boldsymbol{d}}(k)=\left[\begin{array}{c}x_{d 1}(k) \\ x_{d 2}(k) \\ \ldots \\ x_{d N-1}(k) \\ x_{d N}(k)\end{array}\right], \boldsymbol{A}_{\boldsymbol{d}}=\left[\begin{array}{cccccc}0 & 1 & 0 & \ldots & 0 & 0 \\ 0 & 0 & 1 & \ldots & 0 & 0 \\ \ldots & \ldots & \ldots & \ldots & \ldots & \ldots \\ 0 & 0 & 0 & \ldots & 0 & 1 \\ 1 & 0 & 0 & \ldots & 0 & 0\end{array}\right], \boldsymbol{C}_{\boldsymbol{d}}=\left[\begin{array}{llllll}1 & 0 & 0 & \ldots & 0 & 0\end{array}\right]$.

Combining (2) and (3), the extended system state-space equation can be seen in (4).

$$
\left\{\begin{array}{c}
{\left[\begin{array}{c}
\boldsymbol{x}(k+1) \\
\boldsymbol{x}_{\boldsymbol{d}}(k+1)
\end{array}\right]=\left[\begin{array}{cc}
\boldsymbol{A} & \boldsymbol{E} \boldsymbol{C}_{\boldsymbol{d}} \\
\mathbf{0} & \boldsymbol{A}_{\boldsymbol{d}}
\end{array}\right]\left[\begin{array}{c}
\boldsymbol{x}(k) \\
\boldsymbol{x}_{\boldsymbol{d}}(k)
\end{array}\right]+\left[\begin{array}{l}
\boldsymbol{B} \\
\boldsymbol{o}
\end{array}\right] R(k)} \\
V(k)=\left[\begin{array}{ll}
\boldsymbol{C} & \mathbf{0}
\end{array}\right]\left[\begin{array}{c}
\boldsymbol{x}(k) \\
\boldsymbol{x}_{\boldsymbol{d}}(k)
\end{array}\right]
\end{array}\right.
$$

Since the states in $\boldsymbol{x}$ are all known or measurable, the disturbance states $\boldsymbol{x}_{\boldsymbol{d}}$ can be observed by the reducedorder observer as in (5).

$$
\begin{aligned}
& \widehat{\boldsymbol{x}}_{\boldsymbol{d}}(k+1)=\left[\boldsymbol{x}(k+1)-\boldsymbol{A} \boldsymbol{x}(k)-\boldsymbol{B} R(k)-\boldsymbol{E} \boldsymbol{C}_{\boldsymbol{d}} \widehat{\boldsymbol{x}}_{\boldsymbol{d}}(k)\right] \overline{\boldsymbol{A}}_{\boldsymbol{d}} \widehat{\boldsymbol{x}}_{\boldsymbol{d}}(k)+\boldsymbol{L} \\
& =\left(\overline{\boldsymbol{A}}_{\boldsymbol{d}}-\boldsymbol{L} \boldsymbol{E} \boldsymbol{C}_{\boldsymbol{d}}\right) \widehat{\boldsymbol{x}}_{\boldsymbol{d}}(k)+\boldsymbol{L}[\boldsymbol{x}(k+1)-\boldsymbol{A} \boldsymbol{x}(k)-\boldsymbol{B} R(k)] \\
& =\left(\overline{\boldsymbol{A}}_{\boldsymbol{d}}-\boldsymbol{L}_{\boldsymbol{d}} b_{1} \boldsymbol{C}_{\boldsymbol{d}}\right) \widehat{\boldsymbol{x}}_{\boldsymbol{d}}(k)+\boldsymbol{L}_{\boldsymbol{d}}\left[x_{2}(k+1)-b_{1} k_{i} x_{1}(k)-\left(a_{1}-b_{1} k_{p}\right) x_{2}(k)-b_{1} k_{p} R(k)\right]
\end{aligned}
$$




$$
=\left(\overline{\boldsymbol{A}}_{\boldsymbol{d}}-\boldsymbol{L}_{\boldsymbol{d}} b_{1} \boldsymbol{C}_{\boldsymbol{d}}\right) \widehat{\boldsymbol{x}}_{\boldsymbol{d}}(k)+\boldsymbol{L}_{\boldsymbol{d}}\left[x_{2}(k+1)-a_{1} x_{2}(k)-b_{1} U(k)\right] \rightarrow \text { as in Fig. } 1
$$

where, $\boldsymbol{L}=\left[\begin{array}{ll}\mathbf{0} & \boldsymbol{L}_{\boldsymbol{d}}\end{array}\right]=\left[\begin{array}{cc}0 & L_{1} \\ 0 & L_{2} \\ 0 & \ldots \\ 0 & L_{N-1} \\ 0 & L_{N}\end{array}\right], \overline{\boldsymbol{A}}_{\boldsymbol{d}}=\left[\begin{array}{cccccc}0 & 1 & 0 & \ldots & 0 & 0 \\ 0 & 0 & 1 & \ldots & 0 & 0 \\ \ldots & \ldots & \ldots & \ldots & \ldots & \ldots \\ 0 & 0 & 0 & \ldots & 0 & 1 \\ Q & 0 & 0 & \ldots & 0 & 0\end{array}\right]$.

The $\boldsymbol{L}$ matrix is the observer gain matrix. The $Q$ in the matrix $\overline{\boldsymbol{A}_{\boldsymbol{d}}}$ is known as the forgetting factor, which is a value between zero and one. It is not used in the DO, but commonly used in the RC to tune the magnitude response of the disturbance.

Assuming the observed disturbance is added into the system as shown in Fig.1, where, $\boldsymbol{K}_{\boldsymbol{d}}$ can be calculated from (6).

$$
U_{r o}=\boldsymbol{K}_{\boldsymbol{d}} \widehat{\boldsymbol{x}}_{\boldsymbol{d}}=D=x_{d 1} \Rightarrow \boldsymbol{K}_{\boldsymbol{d}}=\left[\begin{array}{llllll}
1 & 0 & 0 & \ldots & 0 & 0
\end{array}\right], \quad \boldsymbol{K}_{\boldsymbol{d}} \widehat{\boldsymbol{x}}_{\boldsymbol{d}}=\hat{x}_{d 1}
$$

The ultimate state-space equation for the whole control system becomes (7).

$$
\left\{\begin{array}{c}
{\left[\begin{array}{c}
\boldsymbol{x}(k+1) \\
\boldsymbol{x}_{\boldsymbol{d}}(k+1) \\
\widehat{\boldsymbol{x}}_{\boldsymbol{d}}(k+1)
\end{array}\right]=\left[\begin{array}{ccc}
\boldsymbol{A} & \boldsymbol{E} \boldsymbol{C}_{\boldsymbol{d}} & -\boldsymbol{K}_{\boldsymbol{d}} \\
\mathbf{0} & \boldsymbol{A}_{\boldsymbol{d}} & \mathbf{0} \\
\mathbf{0} & \boldsymbol{L} \boldsymbol{C}_{\boldsymbol{d}} & \overline{\boldsymbol{A}}_{\boldsymbol{d}}-\boldsymbol{L E} \boldsymbol{C}_{\boldsymbol{d}}
\end{array}\right]\left[\begin{array}{c}
\boldsymbol{x}(k) \\
\boldsymbol{x}_{\boldsymbol{d}}(k) \\
\widehat{\boldsymbol{x}}_{\boldsymbol{d}}(k)
\end{array}\right]+\left[\begin{array}{l}
\boldsymbol{B} \\
\boldsymbol{o}
\end{array}\right] R(k)} \\
V(k)=\left[\begin{array}{lll}
\boldsymbol{C} & \mathbf{0} & \mathbf{0}
\end{array}\right]\left[\begin{array}{c}
\boldsymbol{x}(k) \\
\boldsymbol{x}_{\boldsymbol{d}}(k) \\
\boldsymbol{x}_{\boldsymbol{d}}(k)
\end{array}\right]
\end{array}\right.
$$

The system closed loop poles can be obtained by calculating the eigen values of the system matrix in (8).

$$
\left|\lambda I_{2 N+2}-\left[\begin{array}{ccc}
A & E C_{d} & -K_{d} \\
0 & A_{d} & 0 \\
0 & L C_{d} & \bar{A}_{d}-L E C_{d}
\end{array}\right]\right|=\left|\lambda I_{2}-A\right|\left|\lambda I_{N}-A_{d}\right|\left|\lambda I_{N}-\left(\bar{A}_{d}-L E C_{d}\right)\right|
$$

where, $\boldsymbol{I}_{N}$ denotes the unity matrix of size $N$.

As shown in (8), the eigen values of the system consist of the eigen values of $\boldsymbol{A}, \boldsymbol{A}_{\boldsymbol{d}}$, and $\left(\overline{\boldsymbol{A}}_{\boldsymbol{d}}-\boldsymbol{L} \boldsymbol{E} \boldsymbol{C}_{\boldsymbol{d}}\right)$, which implies that the RO and the PI controller can be designed independently.

In summary, although there are some differences between the RO and DO (i.e. the RO is a $\mathrm{N}$-dimension observer, whereas the DO is a first-order observer, and the forgetting faction included in the RO is not present in the DO), the separation principle of the DO is still valid for the RO. This makes the RO easier to tune than the RC, since it can be added to any existing loop without interfering with the system stability, whereas the RC may cause instability.

\section{Performance Analysis in Simulation}

The system stability can be guaranteed simply by making the feedback controller and the RO stable separately. The next question is how the system performance can be optimised. In this section, the influences of the observer dimension $N$, the observer gain matrix $\boldsymbol{L}_{\boldsymbol{d}}$, and the forgetting factor $Q$ will be discussed by comparing output waveforms (currents and speed) acquired with different control parameter settings. The settings for the PI and the RO that provide the best performance will also be analysed.

A simulation model has been built in Matlab/Simulink for the disturbance torque reduction of the PMSM drive. The simulation model includes a high bandwidth deadbeat current loop[10], a PMSM model with Finite Element (FE) analysis-generated lookup tables for magnetic flux and cogging torque (of which the main ripple components are the $6^{\text {th }}, 12^{\text {th }}$, and $18^{\text {th }}$ harmonics with respect to electrical frequency), a speed 
loop and RO as in Fig.1. Because the deadbeat current controller do not contain a integer, offsets in current response may occur due to voltage drops on the inverter and electrical parameter mismatches. Periodic errors in the current response also presents due to harmonics from the inverter. However, such errors can all be recognised as torque disturbances and therefore can be handled by the RO. For this application, the state $D$ in Fig. 1 represents the load torque, the disturbance torque and the equivalent disturbance torque caused by current control errors, the input $R$ represents the reference speed, and the output $V$ represents the speed feedback. The number of pole pairs is 3 . Stator resistance $R_{s}$ is $1.4 \Omega$, dq-axis inductances are $4.8 \mathrm{mH}$ and $7.1 \mathrm{mH}$ respectively, moment of inertia $J$ and friction factor $B$ are $9 \mathrm{kgcm}^{2}$ and $0.004 \mathrm{Nms} / \mathrm{rad}$.

\section{The choice of $N$}

The observer dimension $N$ can be calculated as $N=f_{s} / f_{d}$. Where, $f_{d}$ should be the fundamental frequency of the periodic disturbance. In PMSM drives, the fundamental frequency of the periodic disturbance is the rotational frequency. For example, at $1500 \mathrm{rpm}, f_{d}=25 \mathrm{~Hz}$. Assuming $f_{s}=10 \mathrm{kHz}$, then $N=400$. It is worth pointing out that the normal RO does not support variable speed conditions when $N$ varies online or is a non-integer. An angle-based RO has been proposed in [8] for variable speed, and the performance in speed transients will not be discussed in this paper.

\section{The choice of $L_{d}$ and $Q$}

The eigenvalues of matrix $\left(\overline{\boldsymbol{A}}_{\boldsymbol{d}}-\boldsymbol{L} \boldsymbol{E} \boldsymbol{C}_{\boldsymbol{d}}\right)$ can be calculated by solving equation (9).

$$
\left|\lambda \boldsymbol{I}_{\boldsymbol{N}}-\left(\overline{\boldsymbol{A}}_{\boldsymbol{d}}-\boldsymbol{L} \boldsymbol{E} \boldsymbol{C}_{\boldsymbol{d}}\right)\right|=\lambda^{N}+b_{1} L_{1} \lambda^{N-1}+b_{1} L_{2} \lambda^{N-2}+\cdots+b_{1} L_{N-1} \lambda+b_{1} L_{N}-Q=0
$$

It is noticed from (9) that the easiest way of placing the poles is to set $L_{1}=L_{2}=\ldots=L_{N-1}=0$, such that the poles are determined by just two parameters, i.e. $L_{N}$ and Q. For stability reason, $\left|Q-b_{1} L_{N}\right|<1$ must hold.

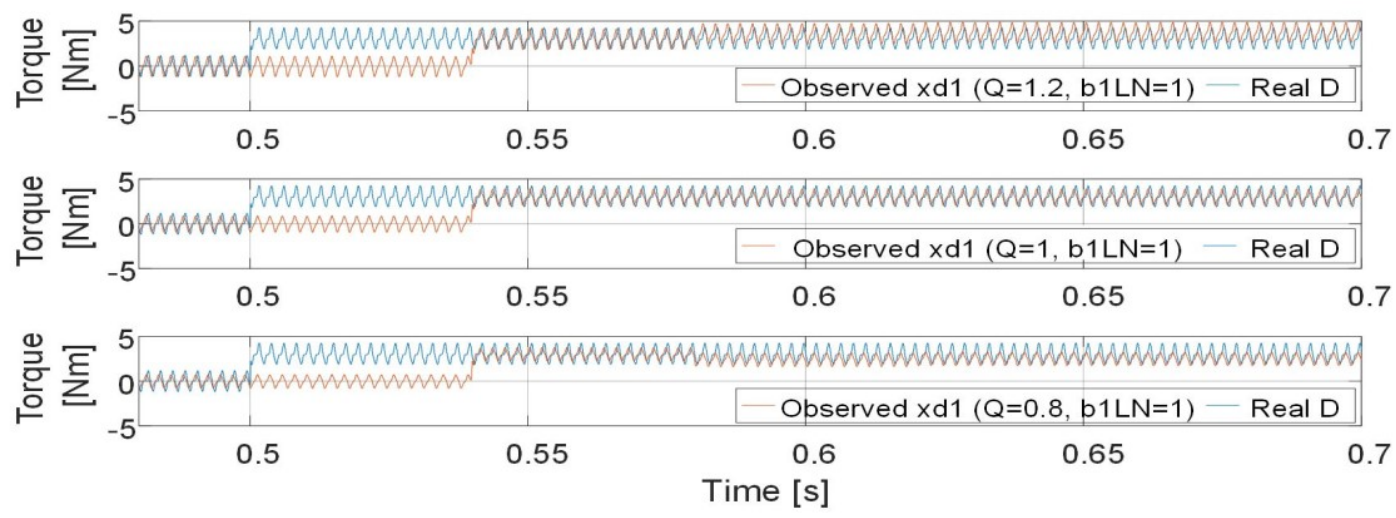

Fig.3 Observed and real disturbance during a disturbance step with different $Q$ in simulation.

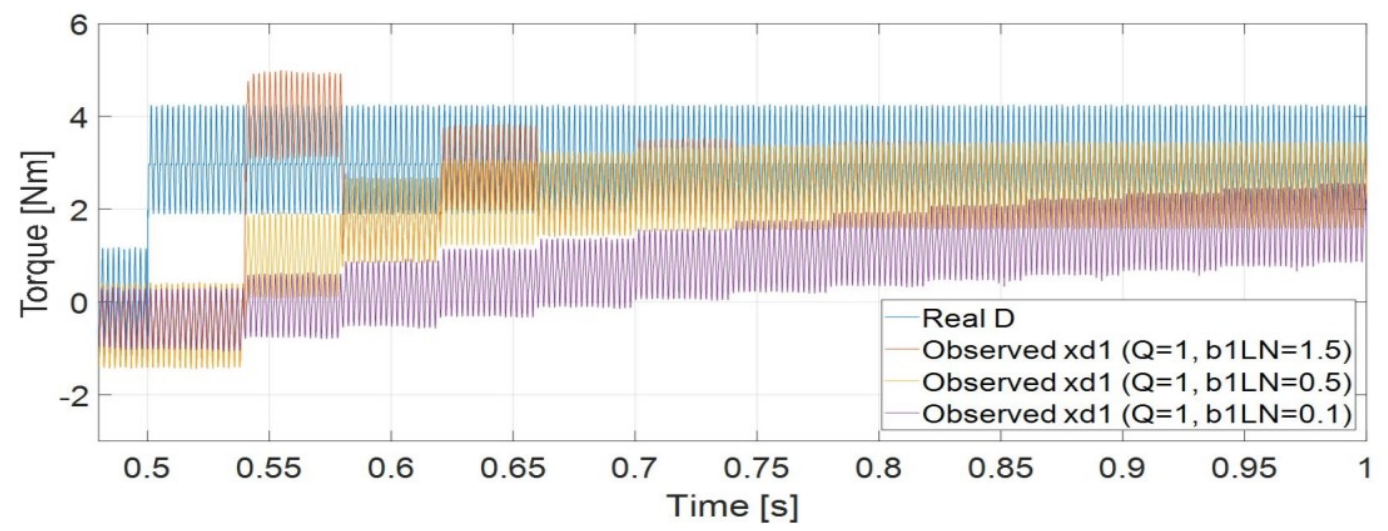

Fig.4 Observed and real disturbance during a disturbance step with different $b_{I} L_{N}$ in simulation. 
Fig. 3 and Fig. 4 show the observed disturbance $\hat{x}_{d l}$ and the actual disturbance $D$ with different values of $Q$ and $L_{N}$, respectively. The RO is running on its own without connecting its output to the system, and a $3 \mathrm{Nm}$ load torque step is applied at $0.5 \mathrm{~s}$. The results show that the observation is the most accurate when $Q=1$. And the value of $L_{N}$ does not affect the observed disturbance value at steady state but affects mainly the convergence speed.

\section{The interaction between PI and RO}

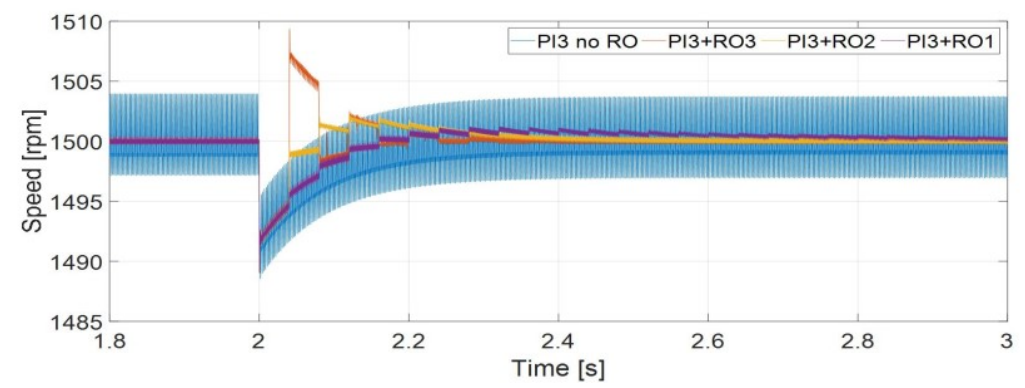

(a) $x_{2}$

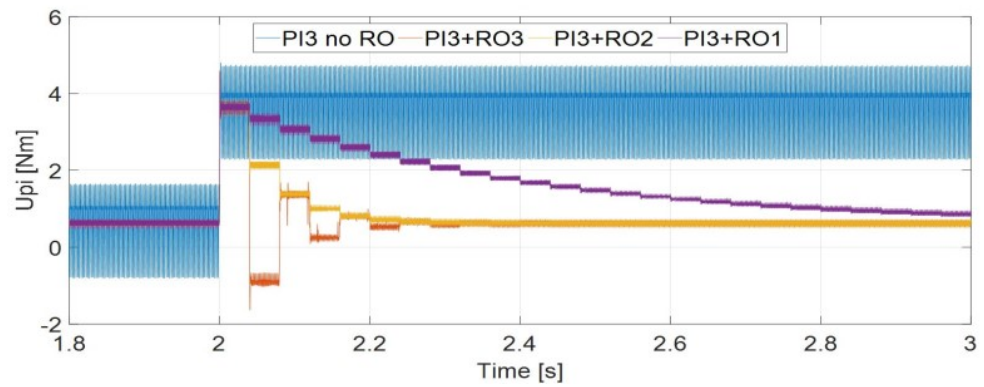

(b) $U_{p i}$

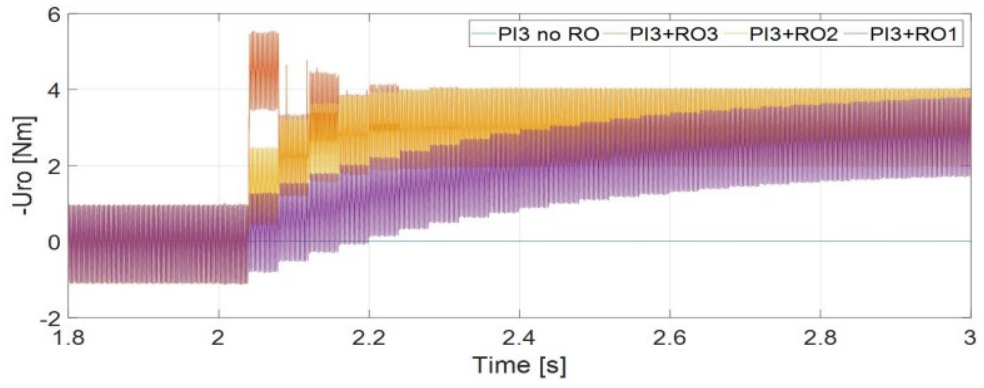

(c) $-U_{r o}$

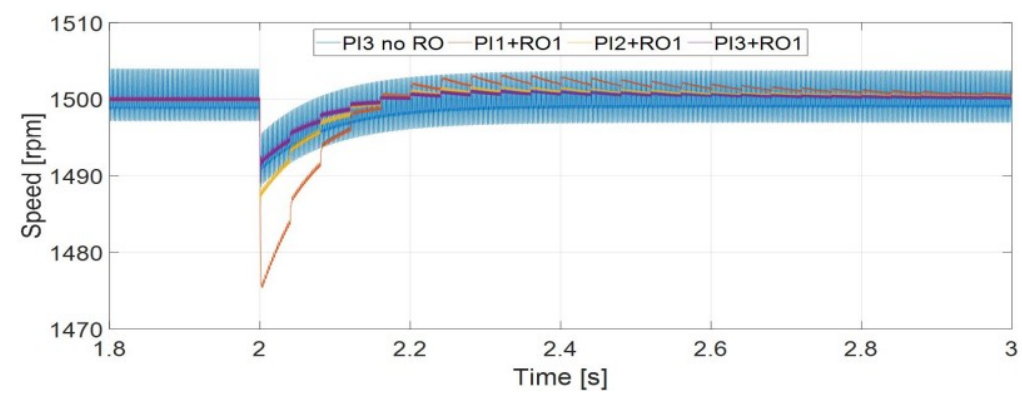

(d) $x_{2}$

Fig.5 Speed and torque references during a disturbance step with different PI and RO settings in simulation. 
Fig.5 shows the speed responses and torque references under different combinations of PI and RO tuned to be slow, medium and fast. The three PIs compared are named as PI1, PI2, and PI3, with which the speed loop bandwidths are $200 \mathrm{~Hz}, 400 \mathrm{~Hz}$, and $600 \mathrm{~Hz}$, respectively. The three ROs compared are called RO1, RO2, RO3, whose gains $L_{N}$ are set to be $0.1 / b_{1}, 0.5 / b_{1}, 1.5 / b_{1}$, respectively. Define the output of PI as $U_{p i}$ and the torque reference generated by RO as $U_{r o}$, where $U_{r o}=\mathbf{K}_{\mathbf{d}} \hat{\mathbf{x}}_{\mathbf{d}}$. According to Fig.1, the torque reference $U=U_{p i}-U_{r o}$.

Results show that when a step in disturbance is applied suddenly at $2 \mathrm{~s}$, both PI and RO generate the extra torque references but in different pace. PI responses immediately, RO responses after one mechanical rotation. During such period, PI interacts with RO to ensure that the overall torque reference $U$ follows the load. When RO is fast, such interaction deteriorates the smoothness of speed during sudden disturbance steps. Hence, it is suggested to tune PI independently according to the dynamic requirement, while set the gain $L_{N}$ of RO small to minimize the interaction between PI and RO.

\section{EXPERIMENTAL VALIDATION}

Experimental tests are carried out on the rig shown in Fig.6, where, the control algorithms are implemented using uCube, a high performance DSP/FPGA platform[11]. The machine parameters are shown in Table I. Several practical issues, such as the noise in the position measurement, the compensation of delay in speed feedback, the misidentified mechanical parameters, and the application of angle-based RO under variable speed have been considered in the implementation. The details have been discussed in [8]. As a result, the RO has been implemented in the angle-domain, such that it is adaptive to variable speed with fixed $N . N$ is set to 200. Sampling frequency is $10 \mathrm{kHz}$.

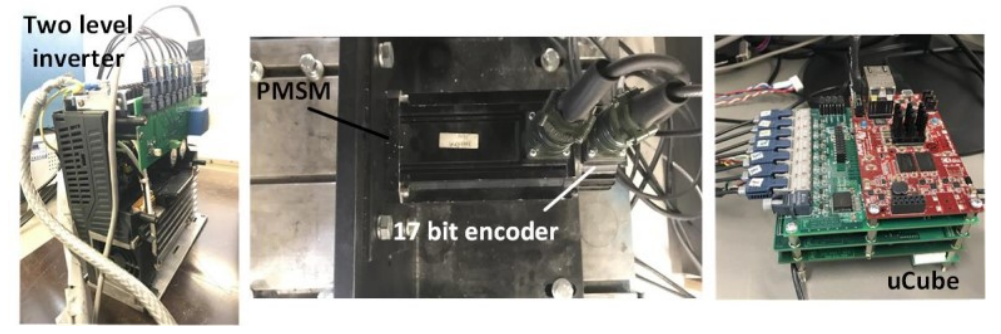

Fig.6 Experimental rig.

Table I Parameters of PMSM

\begin{tabular}{|c|c|c|c|c|c|}
\hline Parameters & Values & Parameters & Values & Parameters & Values \\
\hline $\begin{array}{c}\text { Stator } \\
\text { resistance }\end{array}$ & $0.5 \Omega$ & Pole pairs & 5 & Rated torque & $3.8 \mathrm{Nm}$ \\
\hline $\begin{array}{c}\text { d-axis } \\
\text { inductance }\end{array}$ & $1.667 \mathrm{mH}$ & $\begin{array}{c}\text { Moment of } \\
\text { inertia } J\end{array}$ & $5.52 \mathrm{kgcm}^{2}$ & $\begin{array}{c}\text { Rated current } \\
(\mathrm{rms})\end{array}$ & $5.37 \mathrm{~A}$ \\
\hline $\begin{array}{c}\text { q-axis } \\
\text { inductance }\end{array}$ & $2.468 \mathrm{mH}$ & Friction $B$ & $\begin{array}{c}0.00217 \\
\mathrm{Nms} / \mathrm{rad}\end{array}$ & \\
\hline
\end{tabular}

Since the torque ripple problem is more serious at low speed. The tests have been carried out at $100 \mathrm{rpm}$. To verify the arguments in "The choice of $\mathbf{L}_{\mathbf{d}}$ and $Q$ " section, the observed disturbance when RO is just activated at $0.25 \mathrm{~s}$ and the observed disturbance at steady state are compared in Fig.7 and Fig.8, where, the observed disturbance at steady state is considered as the real disturbance. Ideally, PI is the faster, the better, but practically, using a faster PI will increase the audible noise due to quantization noise in the position 
feedback. Hence, during the test, the PI is tuned to provide $200 \mathrm{~Hz}$ speed loop bandwidth, and RO is running on its own without connecting its output to the system.

In Fig.7, gain $L_{N}=1 / b_{1}$, and $Q$ varies from 0.5 to 1.5 . The results confirm the fact that $Q=1$ is the best setting. In Fig.8, $Q$ is fixed to 1 , while $L_{N}$ varies from $0.005 / b_{1}$ to $1.5 / b_{1}$. The results are like the simulation results in Fig.4. Overall, again, it is recommended to choose $Q=1$ and $L_{N}$ to a small value. Although a smaller gain $L_{N}$ may slow down the learning process of RO, it does not affect the steady state performance as shown in
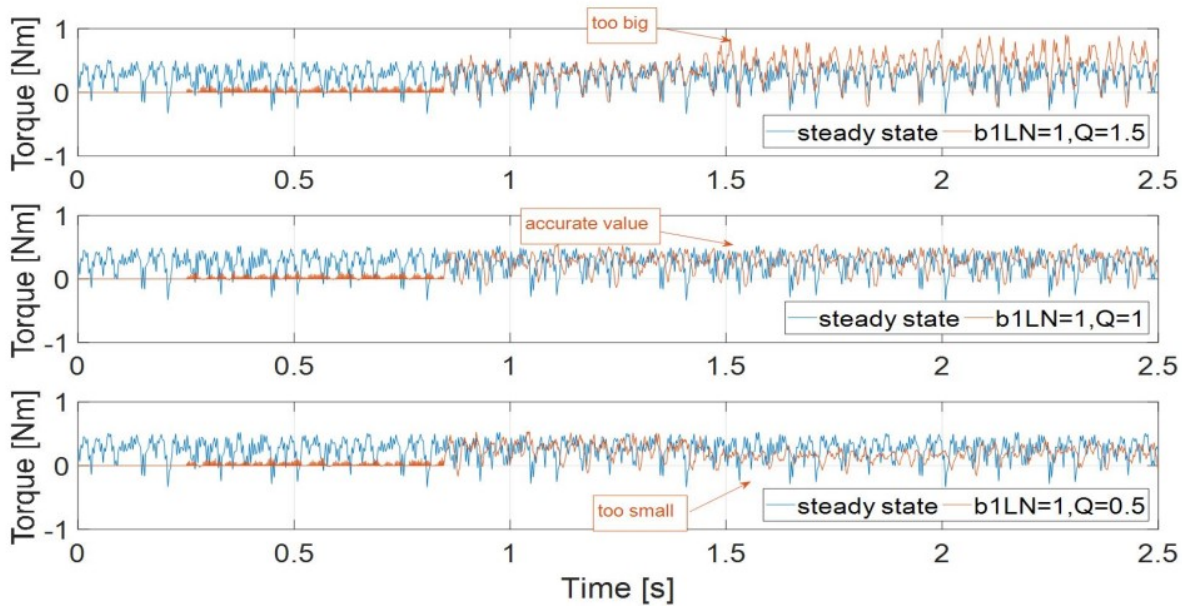

Fig.7 Observed disturbance when just activated and at steady state with different $Q$ in experimental tests with no load.

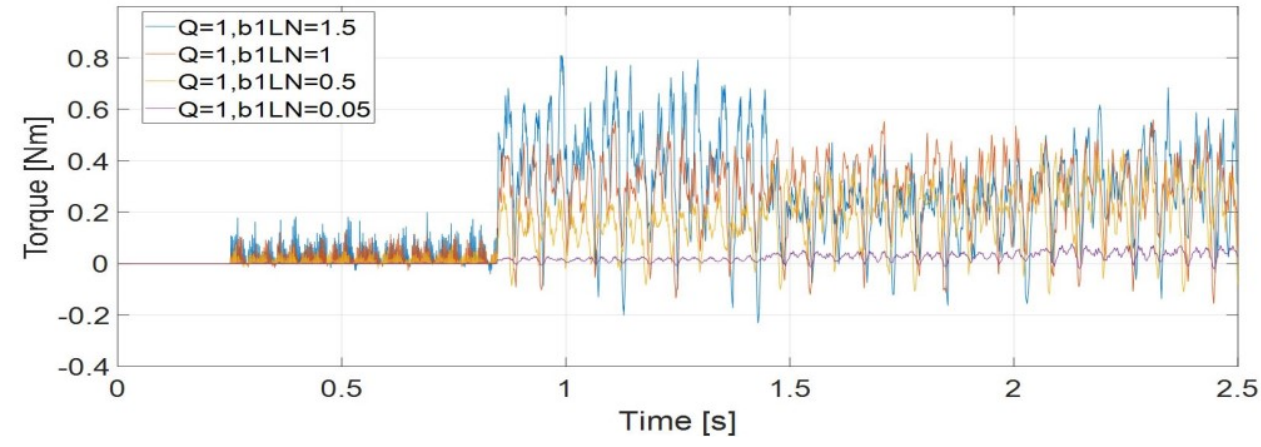

Fig.8 Observed disturbance when just activated and at steady state with different $b_{I} L_{N}$ in experimental tests with no load.

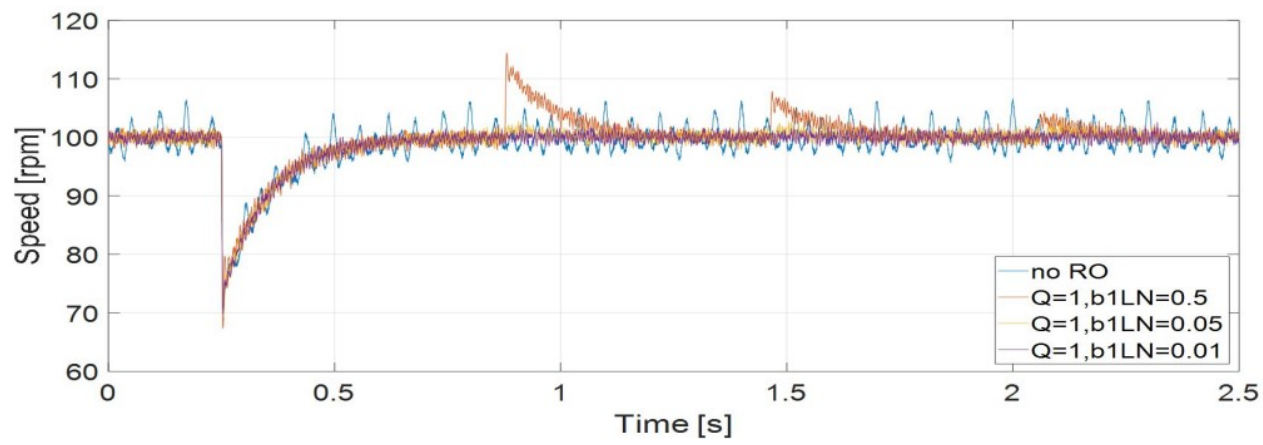

Fig.9 Speed during a disturbance step in experimental tests (a $4 \mathrm{~A}$ current disturbance is applied at $0.25 \mathrm{~s}$ ). 
Fig.9, where the speed ripple has been reduced to the same level with $L_{N}=0.5 / b_{1}$ and $L_{N}=0.01 / b_{1}$. A disturbance step in current is applied at $0.25 \mathrm{~s}$ in Fig.9, and same trend as in Fig. 5 can be seen in Fig.9 that using a smaller $L_{N}$ is clearly the better.

\section{CONCLUSION}

The RO is a powerful tool to reduce periodic disturbance in power electronics and drives systems. It is obtained as a combination of the DO and the RC, such that it is easier to tune than the RC and more powerful in reducing periodic disturbance than the DO. A systematic tuning method for the RO, however, has not yet been fully discussed in literature. This paper aims to prove mathematically that the separation principle of the DO still applies to the RO, and to investigate how the RO and the feedback controller can be tuned together to provide the best performance. It is a very promising technology that can directly be added as a plug in feature for many applications where periodic disturbance is an issue. Overall, the feedback controller can be designed independently according to the dynamic requirements, and it is suggested to set the $Q$ parameter of RO to one and keep its gain $L_{N}$ a small to reduce the interaction between the feedback controller and $\mathrm{RO}$, and to achieve a smoother transient behaviour.

[1] A. Lidozzi, C. Ji, L. Solero, P. Zanchetta, and F. Crescimbini, "Resonant-Repetitive Combined Control for Stand-Alone Power Supply Units," IEEE Transactions on Industry Applications, vol. 51, no. 6, pp. 46534663, 2015.

[2] M. Tang, A. Gaeta, A. Formentini, and P. Zanchetta, "A Fractional Delay Variable Frequency Repetitive Control for Torque Ripple Reduction in PMSMs," IEEE Transactions on Industry Applications, vol. 53, no. 6, pp. 5553-5562, 2017.

[3] Y. Seki, K. Ohishi, Y. Yokokura, Y. Ide, and D. Kuraishi, "Quick torque ripple suppression controlled fast position servo based on hybrid system of disturbance observer and torque ripple table," in IECON 2015 - 41st Annual Conference of the IEEE Industrial Electronics Society, 2015, pp. 000817-000822.

[4] H. Muramatsu and S. Katsura, "An Adaptive Periodic-Disturbance Observer for Periodic-Disturbance Suppression," IEEE Transactions on Industrial Informatics, vol. 14, no. 10, pp. 4446-4456, 2018.

[5] W. Chen, J. Yang, L. Guo, and S. Li, "Disturbance-Observer-Based Control and Related Methods-An Overview," IEEE Transactions on Industrial Electronics, vol. 63, no. 2, pp. 1083-1095, 2016.

[6] T. Senjyu, H. Kamifurutono, and K. Uezato, "Robust current control method with disturbance voltage observer for voltage source PWM inverter," in Proceedings of 1995 International Conference on Power Electronics and Drive Systems. PEDS 95, 1995, pp. 379-384 vol.1.

[7] M. Tang, A. Formentini, S. Odhano, and P. Zanchetta, "Design of a repetitive controller as a feed-forward disturbance observer," in IECON 2016 - 42nd Annual Conference of the IEEE Industrial Electronics Society, 2016, pp. 78-83.

[8] M. Tang, A. Formentini, S. A. Odhano, and P. Zanchetta, "Torque Ripple Reduction of PMSMs using a Novel Angle-based Repetitive Observer," IEEE Transactions on Industrial Electronics, pp. 1-1, 2019.

[9] M. D. Benedetto, A. Lidozzi, L. Solero, M. Tang, A. Formentini, and P. Zanchetta, "Disturbance-Observer Assisted Controller for Stand-Alone Four-Leg Voltage Source Inverter," in 2018 IEEE Energy Conversion Congress and Exposition (ECCE), 2018, pp. 2265-2270.

[10] M. Tang, A. Gaeta, A. Formentini, K. Ohyama, P. Zanchetta, and G. Asher, "Enhanced DBCC for high-speed permanent magnet synchronous motor drives," IET Power Electronics, vol. 9, no. 15, pp. 2880-2890, 2016.

[11] A. Galassini, G. L. Calzo, A. Formentini, C. Gerada, P. Zanchetta, and A. Costabeber, "uCube: Control platform for power electronics," in 2017 IEEE Workshop on Electrical Machines Design, Control and Diagnosis (WEMDCD), 2017, pp. 216-221: IEEE. 